\title{
Role of Balloon-Sheathed Intraductal Ultrasonography for Patients with Extensive Pneumobilia
}

\author{
Ha-Na Kim*, Chang-Hwan Park*, Eun-Ae Cho*, Soo-Jung Rew ${ }^{\dagger}$, In-Hyung Park*, Sung-Uk Lim*, Chung-Hwan Jun*, Seon- \\ Young Park*, Hyun-Soo Kim*, and Sung-Kyu Choi* \\ ${ }^{*}$ Department of Internal Medicine, Chonnam National University Medical School, and ${ }^{\dagger}$ Department of Internal Medicine, Gwangju Chritian \\ Hospital, Gwangju, Korea
}

Intraductal ultrasonography (IDUS) is one of the most useful diagnostic tools for various extrahepatic biliary diseases. However, conventional IDUS has some limitations in providing accurate cross-sectional imaging of the bile duct in patients with extensive pneumobilia. Using a balloonsheathed catheter, the US system (balloon-sheathed IDUS) can overcome these limitations. Sixteen patients underwent balloon-sheathed IDUS during endoscopic retrograde cholangiography. The balloon-sheathed IDUS was inserted via a transpapillary route when visualization of the bile duct with conventional IDUS was distorted by extensive pneumobilia. The patient group had a mean age of 65.5 years, and $56.3 \%$ (9/16) were male. The balloon-sheathed IDUS permitted successful visualization of the bile duct in all patients, regardless of the extent of pneumobilia. Using this system, remnant common bile duct stones were detected in five patients (31.3\%), and cholangiocarcinoma was detected in one patient (6.3\%). The balloon-sheath IDUS aided in stone sweeping. No significant complications, including bleeding, perforation, or pancreatitis, occurred in any of the patients. The balloon-sheathed catheter US system was useful and safe for biliary IDUS in patients with extensive pneumobilia. (Gut Liver 2015;9:561-565)

Key Words: Common bile duct diseases; Intraductal ultrasonography

\section{INTRODUCTION}

Symptomatic recurrent bile duct stones have been reported in $4 \%$ to $24 \%$ of cases after endoscopic retrograde cholangiopancreatography (ERCP). ${ }^{1}$ Small bile duct stones persisting after endoscopic sphincterotomy (ES) and stone extraction may be a nidus for further stone formation. Gall bladder status, lithotripsy, and pneumobilia were significantly related to the risk of bile duct stone recurrence after ES. ${ }^{2}$

Intraductal ultrasonography (IDUS) is a highly sensitive diagnostic modality in extrahepatic biliary diseases. ${ }^{3-5}$ IDUS is more effective in the diagnosis of bile duct stones than ERCP. ${ }^{6}$ However, conventional IDUS has known limitations in providing accurate cross-sectional imaging of the bile duct in patients with pneumobilia. ${ }^{7-11}$ Therefore, the development of a new IDUS system is necessary to overcome these limitations.

\section{CASE REPORT}

From December 2013 to February 2014, 16 patients with pneumobilia underwent IDUS during ERCP, using a balloonsheathed catheter US system. Before the ERCP, all the patients had been diagnosed with common bile duct (CBD) stones by various imaging modalities. Written informed consent was obtained from all patients. We performed this retrospective study in accordance with the guidelines of the Institutional Review Board.

All of the ERCP procedures were performed using a standard side-viewing duodenoscope (TJF-160F; Olympus, Tokyo, Japan) in an endoscopy suite. During ERCP, conventional IDUS with a 2.0-mm-diameter intraductal US probe (UM-G20-29R; Olympus) was performed to detect remnant CBD stones. If visualization of the bile duct using conventional IDUS was distorted by extensive pneumobilia, a balloon-sheathed catheter US system (UM-BS20-26R; Olympus) was used (Fig. 1). The balloon sheath fits over the probe and is assembled in two pieces. A continuous 205-cm catheter with a latex balloon at the tip locks into

Correspondence to: Chang-Hwan Park

Division of Gastroenterology, Department of Internal Medicine, Chonnam National University Hospital, Chonnam National University Medical School, 42 Jebong-ro, Dong-gu, Gwangju 501-757, Korea

Tel: +82-62-220-6296, Fax: +82-62-225-8578, E-mail: p1052ccy@hanmail.net

Received on June 25, 2014. Revised on September 16, 2014. Accepted on October 10, 2014. Published online June 4, 2015 pISSN 1976-2283 eISSN 2005-1212 http://dx.doi.org/10.5009/gnl14231

@ This is an Open Access article distributed under the terms of the Creative Commons Attribution Non-Commercial License (http://creativecommons.org/licenses/by-nc/4.0) which permits unrestricted non-commercial use, distribution, and reproduction in any medium, provided the original work is properly cited. 
a separate, watertight housing. The balloon system can only be used with an endoscope with an accessory channel of $3.4 \mathrm{~mm}$ or larger. The balloon-sheathed catheter US (balloon-sheathed

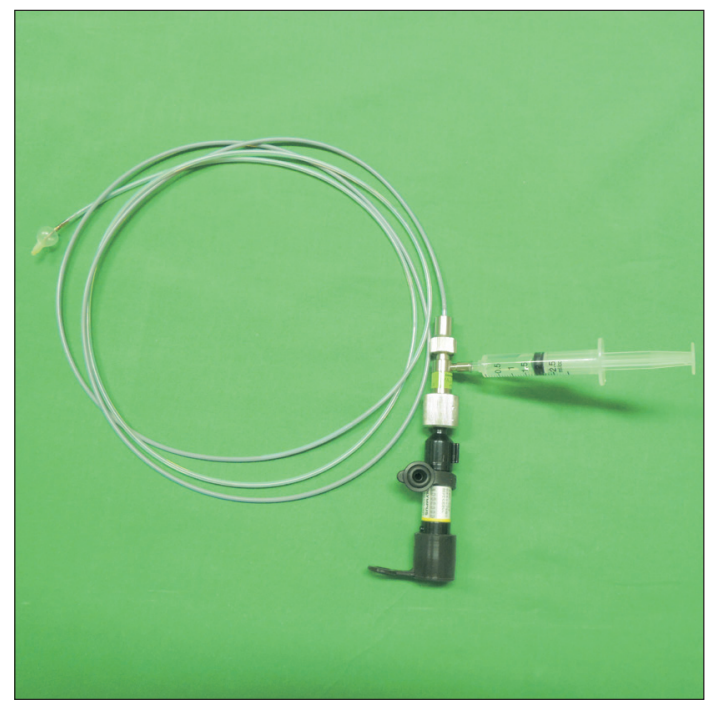

Fig. 1. Balloon-sheathed catheter ultrasonography probe.
IDUS) probe was carefully inserted using slow, short strokes after pulling the syringe plunger to deflate the balloon. While viewing the probe's distal end endoscopically, the probe was advanced to the papilla of Vater (POV) with a proper axis, using up and down manipulation. After inserting the probe via a transpapillary route to the confluent portion of the CBD without a guidewire, we inflated the balloon to the desired diameter by pushing the syringe plunger and turned the three-way stopcock to stop water flow, in order to secure a clean visual field without pneumobilia. Then, the balloon-sheathed IDUS probe was slowly withdrawn to the POV. If CBD stones and sludge were detected, basket extraction was attempted. If a suspected cholangiocarcinoma was discovered using this system, biopsy and brush cytology were carried out for diagnosis.

During the study period, a total of 158 patients underwent biliary IDUS; of these, 16 patients $(16 / 158,10.1 \%)$ also underwent IDUS with the balloon-sheathed catheter US system. The patients had a mean age of 65.5 years (range, 28 to 90 years), and 56.3\% were male. Of the 16 patients, nine (56.3\%) had previously undergone ES; seven (43.7\%) had no prior history of ES. The mean stone size was $7.7 \mathrm{~mm}$ (range, 2.4 to $20 \mathrm{~mm}$ ), and the mean CBD diameter was $14.2 \mathrm{~mm}$ (range, 5 to $23 \mathrm{~mm}$ ).
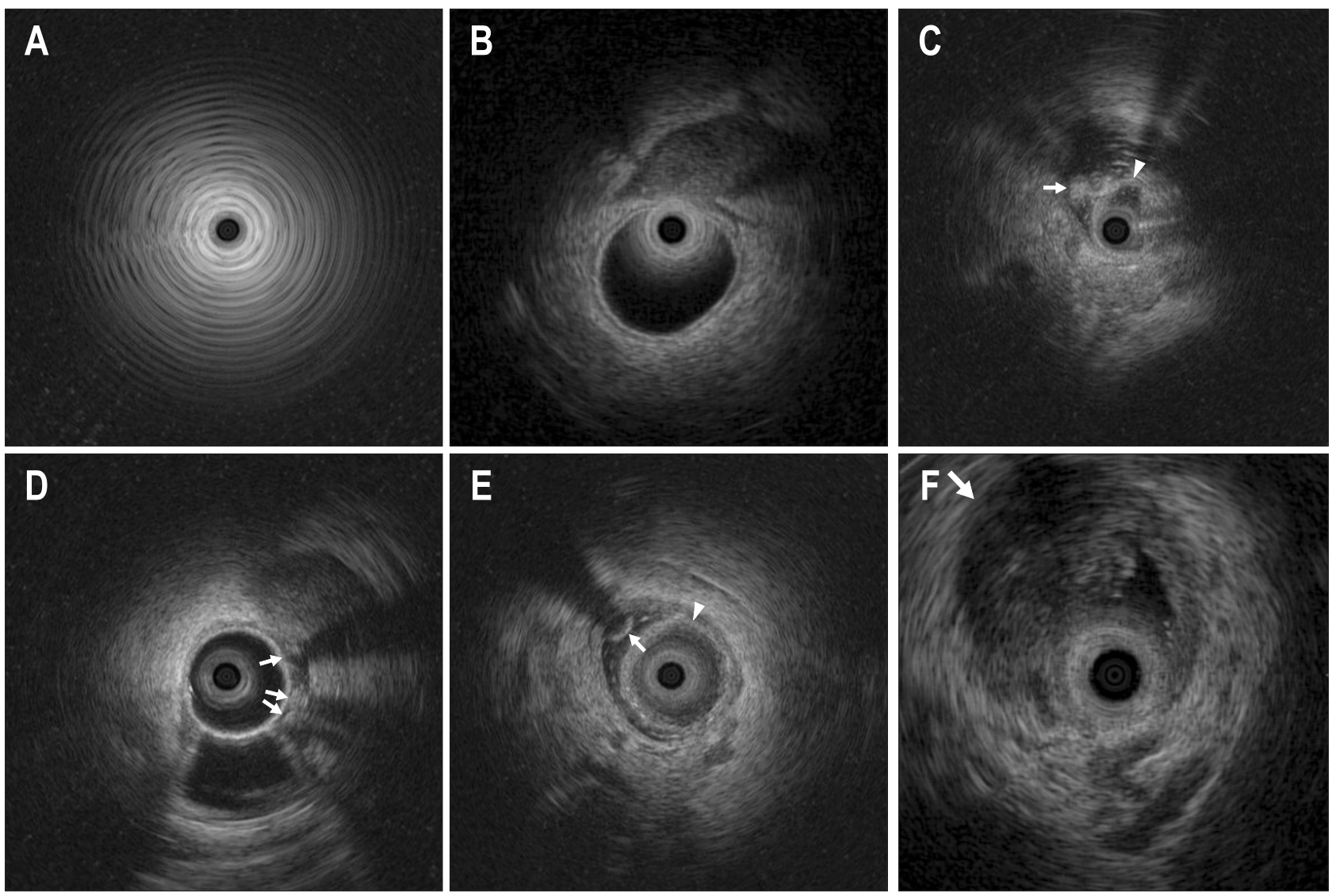

Fig. 2. (A) Extensive pneumobilia as shown by conventional intraductal ultrasonography (IDUS). (B) Extrahepatic bile duct wall (same area in Fig. 2A), visualized with the balloon-sheathed catheter IDUS. (C) Remnant stones (arrow), detected with the balloon (arrowhead)-sheathed catheter IDUS. (D) Remnant stones (arrows), detected with the balloon-sheathed catheter IDUS. (E) Remnant stones (arrow), detected with the balloon (arrowhead)-sheathed catheter IDUS. (F) Cholangiocarcinoma (arrow), detected with the balloon-sheathed catheter IDUS in common bile duct. 
Table 1. Baseline Characteristics and Therapeutic Outcomes

\begin{tabular}{|c|c|c|c|c|c|c|}
\hline Patientno. & Sex/age, yr & Diagnosis & Stone size, mm & Remnant stone & CBD diameter, $\mathrm{mm}$ & Procedure time, min \\
\hline 1 & $\mathrm{M} / 59$ & CBD stones & 15 & No & 23 & 11 \\
\hline 2 & $\mathrm{M} / 55$ & CBD stones & 2.4 & No & 5 & 18 \\
\hline \multirow[t]{2}{*}{3} & $\mathrm{M} / 63$ & CBD stones & 5 & No & 17 & 17 \\
\hline & & langiocarcinc & & & & \\
\hline 4 & $\mathrm{M} / 28$ & CBD stones & 5 & No & 7.5 & 24 \\
\hline 5 & $\mathrm{M} / 69$ & CBD stones & 9 & No & 13 & 40 \\
\hline 6 & $\mathrm{M} / 57$ & CBD stones & 6.6 & No & 18 & 12 \\
\hline 7 & $\mathrm{~F} / 63$ & CBD stones & 9 & Yes & 20 & 11 \\
\hline 8 & $\mathrm{~F} / 66$ & CBD stones & 11.5 & Yes & 15.6 & 16 \\
\hline 9 & $\mathrm{~F} / 84$ & CBD stones & 7.5 & No & 11.5 & 35 \\
\hline 10 & $\mathrm{~F} / 90$ & CBD stones & 6.1 & Yes & 11 & 11 \\
\hline 11 & $\mathrm{~F} / 84$ & CBD stones & 3 & Yes & 20 & 29 \\
\hline 12 & $\mathrm{~F} / 43$ & CBD stones & 4.5 & No & 12 & 20 \\
\hline 13 & $\mathrm{~F} / 88$ & CBD stones & 20 & Yes & 15.2 & 32 \\
\hline 14 & $\mathrm{M} / 79$ & CBD stones & 4 & No & 13 & 17 \\
\hline 15 & $\mathrm{M} / 53$ & CBD stones & 7 & No & 15 & 28 \\
\hline 16 & $\mathrm{M} / 68$ & CBD stones & 7 & No & 10 & 15 \\
\hline
\end{tabular}

CBD, common bile duct; $\mathrm{M}$, male; F, female.

All patients had extensive pneumobilia during ERCP, and the visualization of the bile duct using conventional IDUS was distorted by extensive pneumobilia (Fig. 2A). After the balloonsheathed IDUS probe was inserted, appropriate visualization of the bile duct was achieved in all patients (16/16, 100\%). The baseline characteristics and therapeutic outcomes of the patients are summarized in Table 1. The balloon-sheathed IDUS provided excellent imaging of the bile duct without interference by pneumobilia (Fig. 2B). Using this system, remnant CBD stones were detected in five patients (31.3\%) (Fig. 2C-E). The mean size of the remnant stones was $3.58 \mathrm{~mm}$ (range, 2 to $5 \mathrm{~mm}$ ). In one patient, intraductal ultrasonography showed asymmetrical wall thickening in the mid to distal CBD (Fig. 2F), although abdominal computerized tomography and magnetic resonance cholangiopancreatography images did not show any evidence of cholangiocarcinoma. Biopsy and brush cytology were performed for diagnosis, and well-differentiated adenocarcinoma was confirmed. In some patients, CBD stones were advanced toward the POV with the balloon-sheathed IDUS probe. No significant adverse events, including cholangitis, pancreatitis, or perforation, occurred in any of the patients.

\section{DISCUSSION}

In the present study, balloon-sheathed IDUS was successfully performed in all patients to visualize the bile duct, regardless of the extent of pneumobilia. Using this system, remnant CBD stones were effectively diagnosed, and a case of cholangiocarcinoma was detected. The balloon-sheathed IDUS was also help- ful for stone sweeping, and no significant adverse events were observed. Therefore, balloon-sheathed IDUS can be considered a viable new option for performing biliary IDUS in patients with extensive pneumobilia.

ES and stone extraction are safe and effective in the management of patients with CBD stones. However, the risk of stone recurrence is an important issue for patients and physicians. Many factors are associated with stone recurrence. Of these, the presence of pneumobilia after ES may influence the frequency of stone recurrence. ${ }^{2}$ The mechanism underlying the relationship between pneumobilia and stone recurrence remains unclear. First, pneumobilia permits reflux of the duodenal contents into the bile duct with ease. Loss of function of the biliary sphincter after ES may contribute to duodenobiliary reflux. ${ }^{12}$ Duodenobiliary reflux results in bile infection, which plays an essential role in the formation of brown stones. ${ }^{2}$ Second, the presence of pneumobilia during ERCP makes it difficult to detect residual CBD stones. Therefore, pneumobilia might increase the possibility of residual CBD stones after ES and stone extraction.

Conventional IDUS has been reported to accurately discriminate between stones and pneumobilia, as pneumobilia appears as hyperechoic defects and multiple scattering echoes with strong reverberations with IDUS. ${ }^{1,13,14}$ However, IDUS cannot always distinguish between pneumobilia and bile duct stones, especially in patients with extensive pneumobilia after undergoing ES. ${ }^{7-11}$ All of the patients in our study had considerable pneumobilia in the bile duct after ES. Varadarajulu ${ }^{10}$ suggested that flushing normal saline into the CBD during an IDUS examination improves acoustic coupling and enables the reliable 
differentiation of CBD stones from pneumobilia. However, complete resolution of pneumobilia is not always achieved by flushing with normal saline, especially in patients with a dilated CBD and extensive pneumobilia. In addition, this technique could cause cholangitis and displace small CBD stones into the intrahepatic bile ducts. Therefore, the use of a balloon-sheathed catheter US system was considered for biliary IDUS. In the present study, the balloon-sheathed IDUS proved very useful for the detection of small CBD stones in these patients. Accordingly, the use of a balloon-sheathed IDUS may be able to significantly reduce the number of residual CBD stones.

IDUS after stone extraction may potentially help to detect occult tumors. ${ }^{15}$ Therefore, IDUS after stone extraction can improve the detection rate of early cholangiocarcinoma and thereby patient outcomes. Before ES and stone extraction, IDUS has some difficulty in evaluating the wall of the bile duct behind CBD stones, due to the stones' posterior acoustic shadow. After ES and stone extraction, IDUS can easily evaluate the bile duct wall. However, massive pneumobilia may prevent the clean visualization of the bile duct wall. In the present study, the bile duct was clearly visualized with the balloon-sheathed IDUS, despite extensive pneumobilia. Consequently, a balloon-sheathed IDUS can improve the diagnosis of early cholangiocarcinoma in patients with extensive pneumobilia. It will be necessary to perform a large number of studies to prove the effectiveness of a balloon-sheathed catheter US system in detecting early cholangiocarcinoma.

Although it has great potential, the balloon-sheathed IDUS system has a few limitations. First, the balloon-sheathed IDUS is a wireless system, as it was designed to evaluate subepithelial esophageal lesions. It is difficult to insert the balloon-sheathed catheter via the transpapillary route without ES. Therefore, the development of a guidewire-assisted system is needed. Although probe insertion was feasible without traumatically manipulating the elevator apparatus, it was difficult to insert the probe without the guidewire. For probe insertion without the traumatic manipulation of the elevator apparatus, a therapeutic sideviewing duodenoscope (TJF-160F; Olympus) with a large channel was used for the balloon-sheathed US system. The elevator apparatus was not usually used for inserting the probe. Instead, the axis of the probe was aligned to the same axis of the transpapillary route, after fine manipulation of the bending section of the duodenoscope. Second, the balloon is $10 \mathrm{~mm}$ in size, which is too small to ensure a proper visual field in patients with an extremely dilated CBD and pneumobilia. The development of a larger balloon system will be more effective for examination in cases of extreme dilation. Because the balloon-sheathed IDUS was helpful for stone sweeping in the present study, the development of a strong and variable-sized balloon-sheathed IDUS may be helpful for stone removal. In addition, the development of an ultra-slim IDUS with a 0.035-inch diameter would be useful for IDUS-directed therapeutic ERCP without radiation.
This ultra-slim IDUS can be used to provide real-time ultrasonic cholangiography and can guide simultaneous retrieval of balloons or baskets.

In conclusion, the balloon-sheathed catheter US system was useful and safe for biliary IDUS in patients with extensive pneumobilia and has good potential for future applications. To confirm our study results, larger multicenter studies, including a comparative study between the balloon-sheathed catheter US system and the balloon sweeping method, are needed.

\section{CONFLICTS OF INTEREST}

No potential conflict of interest relevant to this article was reported.

\section{REFERENCES}

1. Ang TL, Teo EK, Fock KM, Lyn Tan JY. Are there roles for intraductal US and saline solution irrigation in ensuring complete clearance of common bile duct stones? Gastrointest Endosc 2009; 69:1276-1281.

2. Ando T, Tsuyuguchi T, Okugawa T, et al. Risk factors for recurrent bile duct stones after endoscopic papillotomy. Gut 2003;52:116121.

3. Chak A, Isenberg G, Kobayashi K, Wong RC, Sivak MV Jr. Prospective evaluation of an over-the-wire catheter US probe. Gastrointest Endosc 2000;51:202-205.

4. Tamada K, Inui K, Menzel J. Intraductal ultrasonography of the bile duct system. Endoscopy 2001;33:878-885.

5. Menzel J, Poremba C, Dietl KH, Domschke W. Preoperative diagnosis of bile duct strictures: comparison of intraductal ultrasonography with conventional endosonography. Scand J Gastroenterol 2000;35:77-82.

6. Lu J, Guo CY, Xu XF, Wang XP, Wan R. Efficacy of intraductal ultrasonography in the diagnosis of non-opaque choledocholith. World J Gastroenterol 2012;18:275-278.

7. Das A, Isenberg G, Wong RC, Sivak MV Jr, Chak A. Wire-guided intraductal US: an adjunct to ERCP in the management of bile duct stones. Gastrointest Endosc 2001;54:31-36.

8. Kubota Y, Takaoka M, Yamamoto S, et al. Diagnosis of common bile duct calculi with intraductal ultrasonography during endoscopic biliary cannulation. J Gastroenterol Hepatol 2002;17:708712.

9. Linghu EQ, Cheng LF, Wang XD, et al. Intraductal ultrasonography and endoscopic retrograde cholangiography in diagnosis of extrahepatic bile duct stones: a comparative study. Hepatobiliary Pancreat Dis Int 2004;3:129-132.

10. Varadarajulu S. A modified technique to overcome the limitations of intraductal ultrasound for diagnosis of common bile duct stones. Endoscopy 2008;40 Suppl 2:E152.

11. Endo T, Ito K, Fujita N, et al. Intraductal ultrasonography in the diagnosis of bile duct stones: when and whom? Dig Endosc 
2011;23:173-175.

12. Bergman JJ, van Berkel AM, Groen AK, et al. Biliary manometry, bacterial characteristics, bile composition, and histologic changes fifteen to seventeen years after endoscopic sphincterotomy. Gastrointest Endosc 1997;45:400-405.

13. Ohashi A, Ueno N, Tamada K, et al. Assessment of residual bile duct stones with use of intraductal US during endoscopic balloon sphincteroplasty: comparison with balloon cholangiography. Gas- trointest Endosc 1999;49(3 Pt 1):328-333.

14. Tsuchiya S, Tsuyuguchi T, Sakai Y, et al. Clinical utility of intraductal US to decrease early recurrence rate of common bile duct stones after endoscopic papillotomy. J Gastroenterol Hepatol 2008;23:1590-1595.

15. Ito Y, Kenmochi T, Egawa T, Hayashi S, Nagashima A, Kitagawa Y. Diagnosis of distal cholangiocarcinoma after the removal of choledocholithiasis. Gastroenterol Res Pract 2012;2012:396869. 